\title{
Allergy testing at OLCHC
}

\author{
Cathryn O'Carroll, John Brady, Joe McNamara, Philip Mayne, Aideen Byrne
}

From Food Allergy and Anaphylaxis Meeting 2014

Dublin, Ireland. 9-11 October 2014

\section{Background}

Allergy is a growing problem in the western world. However, education of health care workers in the field of allergy has not kept up with clinical need. There has been much evidence that medical practitioners use diagnostic tests inaccurately, declaring food allergy where there is none. Removal of food from a patient's diet, especially a child's, has significant nutritional implications that can be irreversible and have a lifelong effect. Furthermore the cost of inappropriate tests is escalating. Guidelines have recently been set indicating that allergy focused history must guide all allergy orders: www.ifan.ie.

\section{Aims of this audit}

1. To optimise the allergy testing service provided for both clinicians and patients.

2. To examine whether allergy testing was in line with current guidelines.

3. To look for areas where cost efficiency could be improved.

4. To gather local data that can be incorporated into education sessions for clinicians.

\section{Methodology}

All sIgE tests ordered through the laboratory at Our Lady's Children's Hospital, Crumlin, from May 8th to Nov 7th 2013, were made available in the form of an excel spread sheet. The outcomes of 4 tests were evaluated, according to age of patient and department from where order originated.

- House Dust Mite sIgE

- Grass sIgE

- Common food panel

- Fruit sIgE

\section{Results}

1. HDM sIgE

- 679 tests were ordered

- $25 \%$ of all tests were ordered on those $<2$ yrs and only $15 / 153(10 \%)$ were positive.

\section{Grass pollen sIgE}

- 638 tests were ordered

- $73 \%$ were negative

Positivity increased with age consistent with all international data.

3. Testing for fruit allergy

- All of these tests were negative

4. The common food panel:

- 519 tests were performed

- $59 \%$ of these were entirely negative

\section{Table 1}

\begin{tabular}{lll}
\hline & Negative & Positive \\
\hline$<1 \mathrm{yr}$ & $77(90 \%)$ & $8(10 \%)$ \\
\hline $1-2 \mathrm{yr}$ & 76 & 7 \\
\hline Average age & $3.8+/-3.6$ & $6.3+/-4.2$ \\
\hline
\end{tabular}

Table 2

\begin{tabular}{lll}
\hline & Negative & Positive \\
\hline Average age & $3.8+/-3.8$ & $7.4+/-4.2$ \\
\hline Median age & $3 \mathrm{yr}$ & $7 \mathrm{yr}$ \\
\hline$<1 \mathrm{yr}$ & $83(100 \%)$ & 0 \\
\hline $1-2 \mathrm{yr}$ & $83(98 \%)$ & 2 \\
\hline
\end{tabular}




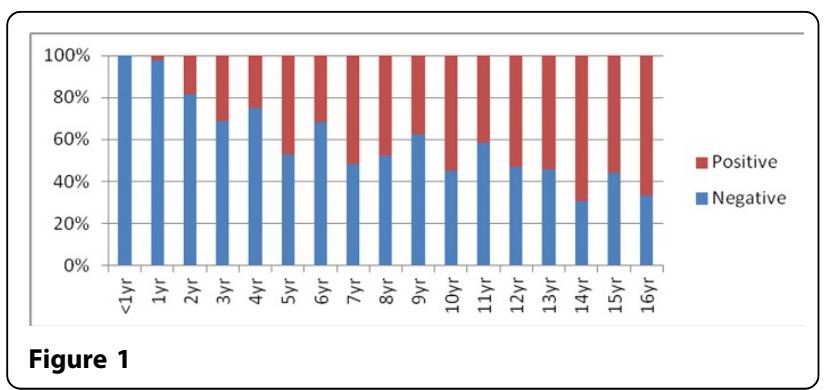

\section{Table 3}

\begin{tabular}{llll}
\hline Food & Total & Positive & Origin \\
\hline Orange & 15 & 0 & $80 \% \mathrm{CPs}$ \\
\hline Strawberry & 11 & 0 & $56 \% \mathrm{CPs}$ \\
\hline
\end{tabular}

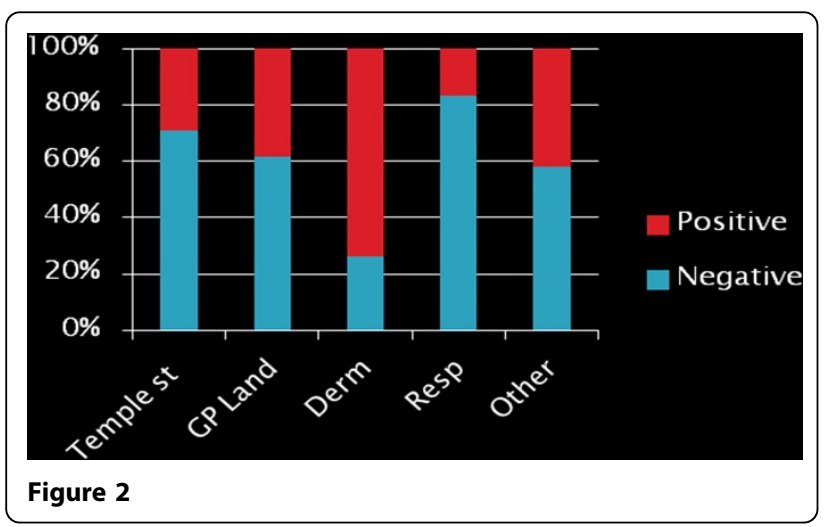

Table 4

\begin{tabular}{|c|c|c|c|}
\hline Conclusions & Recommendations & $\begin{array}{l}\text { Target } \\
\text { date }\end{array}$ & $\begin{array}{l}\text { Responsible } \\
\text { person }\end{array}$ \\
\hline $\begin{array}{l}\text { Clinicians have a poor } \\
\text { understanding of how } \\
\text { to use slgE testing }\end{array}$ & $\begin{array}{l}\text { Formal lecture to } \\
\text { NCHDs }\end{array}$ & $\begin{array}{l}\text { January } \\
2014 \\
\text { (achieved) }\end{array}$ & $\begin{array}{l}\text { Dr Aideen } \\
\text { Byrne }\end{array}$ \\
\hline $\begin{array}{l}\text { Tests are being run } \\
\text { inappropriately with } \\
\text { considerable cost } \\
\text { implications }\end{array}$ & $\begin{array}{l}\text { Establish hospital } \\
\text { protocol for ordering } \\
\text { of IgE tests }\end{array}$ & May 2014 & $\begin{array}{l}\text { Dr Cathryn } \\
\text { O Carroll } \\
\text { Dr Aideen } \\
\text { Byrne }\end{array}$ \\
\hline $\begin{array}{l}\text { Laboratory staff are } \\
\text { unclear how to judge } \\
\text { appropriateness of } \\
\text { test order. }\end{array}$ & $\begin{array}{l}\text { Establish clear } \\
\text { guidelines for } \\
\text { laboratory staff }\end{array}$ & May 2014 & $\begin{array}{l}\text { Joe } \\
\text { McNamara }\end{array}$ \\
\hline $\begin{array}{l}\text { Specific IgE testing for } \\
\text { aeroallergens being } \\
\text { ordered in wrong } \\
\text { population }\end{array}$ & $\begin{array}{l}\text { Orders for slgE to } \\
\text { HDM and Grass } \\
\text { pollen not to be } \\
\text { accepted under } 2 \\
\text { years }\end{array}$ & May 2014 & $\begin{array}{l}\text { Aideen } \\
\text { Byrne Joe } \\
\text { McNamara }\end{array}$ \\
\hline
\end{tabular}

Published: 30 March 2015
doi:10.1186/2045-7022-5-S3-019

Cite this article as: $\mathrm{O}^{\prime} \mathrm{Carroll}$ et al:: Allergy testing at OLCHC. Clinical and

Translational Allergy 2015 5(Suppl 3):O19.

\section{Submit your next manuscript to BioMed Central} and take full advantage of:

- Convenient online submission

- Thorough peer review

- No space constraints or color figure charges

- Immediate publication on acceptance

- Inclusion in PubMed, CAS, Scopus and Google Scholar

- Research which is freely available for redistribution

Submit your manuscript at www.biomedcentral.com/submit 